\title{
Cannabinoid CBI Receptor Immunoreactivity in the Prefrontal Cortex: Comparison of Schizophrenia and Major Depressive Disorder
}

\author{
Stephen M Eggan*,', Samuel R Stoyak', Christopher D Verrico' and David A Lewis ${ }^{1,2}$ \\ 'Department of Psychiatry, University of Pittsburgh, Pittsburgh, PA, USA; 'Department of Neuroscience, University of Pittsburgh, \\ Pittsburgh, PA, USA
}

\begin{abstract}
We recently showed that measures of cannabinoid I receptor (CBIR) mRNA and protein were significantly reduced in dorsolateral prefrontal cortex (DLPFC) area 9 in schizophrenia subjects relative to matched normal comparison subjects. However, other studies have reported unaltered or higher measures of CBIR levels in schizophrenia. To determine whether these discrepancies reflect differences across brain regions or across subject groups (eg, presence of depression, cannabis exposure, etc), we used immunocytochemical techniques to determine whether lower levels of CBIR immunoreactivity are (I) present in another DLPFC region, area 46, in the same subjects with schizophrenia, (2) present in area 46 in a new cohort of schizophrenia subjects, (3) present in major depressive disorder (MDD) subjects, or (4) attributable to factors other than a diagnosis of schizophrenia, including prior cannabis use. CBIR immunoreactivity levels in area 46 were significantly 19\% lower in schizophrenia subjects relative to matched normal comparison subjects, a deficit similar to that observed in area 9 in the same subjects. In a new cohort of subjects, CBIR immunoreactivity levels were significantly 20 and 23\% lower in schizophrenia subjects relative to matched comparison and MDD subjects, respectively. The lower levels of CBIR immunoreactivity in schizophrenia subjects were not explained by other factors such as cannabis use, suicide, or pharmacological treatment. In addition, CBIR immunoreactivity levels were not altered in monkeys chronically exposed to haloperidol. Thus, the lower levels of CBIR immunoreactivity may be common in schizophrenia, conserved across DLPFC regions, not present in MDD, and not attributable to other factors, and thus a reflection of the underlying disease process.
\end{abstract}

Neuropsychopharmacology (2010) 35, 2060-207I; doi:I0.1038/npp.2010.75; published online I6 June 20I0

Keywords: cannabis; cholecystokinin; GABA; GAD 67 ; interneurons; primate; working memory

\section{INTRODUCTION}

Cannabinoid signaling through the cannabinoid 1 receptor (CB1R) mediates the physiological and psychoactive properties of cannabis (Ameri, 1999), and may have an important function in the pathogenesis and/or pathophysiology of schizophrenia (Murray et al, 2007). For example, altered inhibition from $\gamma$-aminobutyric acid (GABA) neurons that express CB1Rs and the neuropeptide cholecystokinin (CCK) may contribute to dorsolateral prefrontal cortex (DLPFC) dysfunction in schizophrenia (Lewis and Sweet, 2009); indeed, measures of CB1R mRNA and protein are reported to be significantly lower in DLPFC area 9 in schizophrenia subjects (Eggan et al, 2008; Uriguen et al, 2009). Furthermore, lower CB1R mRNA expression levels were significantly correlated with those for the mRNAs for

*Correspondence: Dr SM Eggan, Department of Psychiatry, University of Pittsburgh, 381 I O'Hara Street, WI653 BST, Pittsburgh, PA I52। 3, USA, Tel: + | 412624 3934, Fax: + I 4126249910 ,

E-mail: eggansm@upmc.edu

Received 22 January 2010; revised 6 May 2010; accepted 6 May 2010 both $\mathrm{CCK}$ and glutamic acid decarboxylase $\left(\mathrm{GAD}_{67}\right)$, a synthesizing enzyme for GABA (Eggan et al, 2008; Hashimoto et al, 2008a), suggesting that all three transcripts are lower in the same population of DLPFC GABA neurons in schizophrenia.

Alterations in these neurons might contribute to DLPFCmediated cognitive impairments in schizophrenia, such as working memory dysfunction, through the following mechanism (Elvevag and Goldberg, 2000; Weinberger et al, 2001; Lewis et al, 2005). CB1Rs are heavily localized to the axon terminals of CCK-containing, GABA basket neurons (Bodor et al, 2005; Eggan and Lewis, 2007). Activation of CB1Rs inhibits release of GABA from these terminals and strongly suppresses $\mathrm{GABA}_{\mathrm{A}}$ receptormediated inhibitory postsynaptic currents in pyramidal neurons (Trettel et al, 2004; Galarreta et al, 2004; Bodor et al, 2005). Furthermore, systemic administration of CB1R agonists reduces overall levels of GABA in the prefrontal cortex (Pistis et al, 2002). Thus, the lower levels of CB1Rs would be expected to enhance cortical GABA neurotransmission in schizophrenia, providing a compensatory, albeit insufficient, response to the deficit of $\mathrm{GAD}_{67}$-mediated 
GABA synthesis (Eggan et al, 2008), which is thought to contribute to working memory impairments in the illness (Lewis et al, 2005). Activation of CB1Rs by exogenous cannabinoids would be expected to further impair GABA neurotransmission in an already compromised system and produce the exacerbation of symptoms, including impaired working memory, frequently seen in individuals with schizophrenia who use cannabis (D'Souza et al, 2005).

However, in contrast to the findings of lower cortical CB1R levels in schizophrenia (Eggan et al, 2008; Uriguen et al, 2009), other studies have reported unaltered or higher CB1R levels or binding (Dean et al, 2001; Zavitsanou et al, 2004; Newell et al, 2006; Koethe et al, 2007; Deng et al, 2007). These discrepancies might reflect differences in the cortical regions examined (eg, DLPFC area 9, anterior cingulate cortex, posterior cingulate cortex, or superior temporal gyrus), or in the presence of comorbid factors, such as depression, which is present in $\sim 50 \%$ of schizophrenia subjects (Buckley et al, 2009). Interestingly, higher levels of CB1R protein and agonist-stimulated $\left[{ }^{35} \mathrm{~S}\right] \mathrm{GTP} \gamma \mathrm{S}$ binding are present in DLPFC area 9 of major depressive disorder (MDD) subjects (Hungund et al, 2004). Thus, comorbid major depression may confound measures of CB1R levels in schizophrenia subjects. In addition, other factors that may differ across studies, such as the proportion of schizophrenia subjects with cannabis exposure (Linszen et al, 1994; Bersani et al, 2002), may have an impact on the measures of CB1R levels.

To address these issues and to gain a better understanding of the function CB1Rs might have in the pathophysiology of schizophrenia, we used immunocytochemical techniques in postmortem tissue from 26 schizophrenia subjects, 14 MDD subjects, and monkeys chronically exposed to haloperidol or matched control monkeys (four pairs) to determine whether lower levels of CB1R immunoreactivity are (1) common in schizophrenia, (2) present in a previously unstudied DLPFC region, area 46, that is functionally important for working memory processes, and (3) distinctive to schizophrenia or attributable to other factors, such as depression, suicide, and antipsychotic medications, commonly associated with the illness.

\section{MATERIALS AND METHODS}

\section{Human Subject Characteristics}

Following informed consent for brain donation from the next-of-kin using procedures approved by the University of Pittsburgh's Committee for Research Involving the Dead and Institutional Review Board for Biomedical Research, brain specimens were obtained from 63 human subjects during autopsies conducted at the Allegheny County Medical Examiner's Office, Pittsburgh, PA. Two cohorts of non-overlapping schizophrenia subjects were used. The first cohort (Table 1; Supplementary Table S1) consisted of 12 matched pairs of schizophrenia and normal comparison subjects used in our earlier study of CB1R mRNA and protein in DLPFC area 9 (Eggan et al, 2008), for which tissue containing area 46 was available. The second cohort (Table 2; Supplementary Table S2) comprised 14 matched triads of schizophrenia, normal comparison, and MDD subjects. Only two schizophrenia subjects had a secondary axis I diagnosis of depression (640 in cohort 1, Supplementary Table S1; 422 in cohort 2, Supplementary Table S2). To control experimental variance and to reduce biological variance, each subject with schizophrenia was matched for sex and, as closely as possible for age and postmortem interval (PMI), with one normal comparison subject (cohort 1) or one normal comparison subject and one MDD subject (cohort 2). The mean age, PMI, and tissue storage time did not significantly differ across subject groups in either cohort (Tables 1 and 2). Subject characteristics have been previously reported for subjects in cohort 1 (Eggan et al, 2008) and cohort 2 (Volk et al, 2002; Cruz et al, 2009); for diagnostic evaluations and detailed subject characteristics see Supplementary Materials. As most of these subjects were obtained as part of a fixed brain collection (see below), measures of brain $\mathrm{pH}$ could not be obtained; however, brain $\mathrm{pH}$ does not predict stability of immunoreactivity (Beneyto et al, 2009).

\section{Tissue Preparation and Immunocytochemistry}

For each brain, the fresh left hemisphere was cut coronally into $1.0-\mathrm{cm}$ thick blocks, fixed for $48 \mathrm{~h}$ in phosphate

Table I Summary of Cohort I Characteristics

\begin{tabular}{lcccc}
\hline Characteristic & Comparison & Schizophrenia & $\boldsymbol{t}_{\text {-Test }}{ }^{\mathrm{a}}$ & $\boldsymbol{p}$ \\
\hline Sex, M/F, No. & $8 / 4$ & $8 / 4$ & & \\
Race, W/B, No. & $8 / 4$ & $9 / 3$ & & \\
Age, mean (SD), years & $46.3(15.7)$ & $45.3(12.5)$ & 0.57 & 0.58 \\
PMI, mean (SD), hours & $16.5(5.1)$ & $17.5(9.2)$ & -0.59 & 0.57 \\
ST, mean (SD), months & $\mid 16.2(21.4)$ & $119.9(16.3)$ & -0.93 & 0.37 \\
\hline
\end{tabular}

Abbreviations: B, black; F, female; M, male; No., number, PMl; postmortem interval; SD, standard deviation; ST, storage time at $-80^{\circ} \mathrm{C} ; \mathrm{W}$, white. apaired $t$-test, $\mathrm{df}=1 \mathrm{I}$.

Table 2 Summary of Cohort 2 Characteristics

\begin{tabular}{|c|c|c|c|c|c|}
\hline Characteristic & Comparison & Schizophrenia & MDD & ANOVA $F(2,39)$ & $p$ \\
\hline Sex, M/F, No. & $9 / 5$ & $9 / 5$ & $9 / 5$ & & \\
\hline Race, W/B, No. & $10 / 4$ & $10 / 4$ & $13 / 1$ & & \\
\hline Age, mean (SD), years & $53.7(\mid 4.4)$ & $52.9(13.0)$ & $54.0(13.1)$ & 0.03 & 0.98 \\
\hline PMI, mean (SD), hours & I2.8 (5.8) & | $3.5(5.8)$ & $12.2(5.0)$ & 0.21 & 0.81 \\
\hline ST, mean (SD), months & | $82.2(27.8)$ & $190.9(25.0)$ & I $66.2(27.5)$ & 3.07 & 0.06 \\
\hline
\end{tabular}

Abbreviations: B, black; F, female; M, male; No., number; PMl; postmortem interval; SD, standard deviation; ST, storage time at $-80^{\circ} \mathrm{C} ; \mathrm{W}$, white. 
buffered (0.1 M; pH 7.4) $4 \%$ paraformaldehyde at $4^{\circ} \mathrm{C}$, cryoprotected by immersion in solutions of increasing sucrose concentration, and then stored at $-30^{\circ} \mathrm{C}$ in a cryoprotectant solution as described earlier (Eggan and Lewis, 2007). Coronal sections were serially cut on a cryostat $(40 \mu \mathrm{m})$ from tissue blocks containing the DLPFC, and every 10th or 20th section was stained for Nissl substance with thionin to identify the location of area 46 based on cytoarchitectonic criteria (Rajkowska and Goldman-Rakic, 1995; Daviss and Lewis, 1995). For each subject, two sections separated by at least $320 \mu \mathrm{m}$ were chosen; sections from each pair or triad were matched as closely as possible for rostral-caudal level.

Free-floating tissue sections were processed for CB1R immunoreactivity using the avidin-biotin-peroxidase method (Hsu et al, 1981) and 3,3'-diaminobenzidine as described earlier (Eggan and Lewis, 2007; Eggan et al, 2008). The specificity of the affinity-purified rabbit anti-CB1R antibody (anti-CB1R-L15; diluted 1:4000; kindly provided by $\mathrm{Dr}$ Ken Mackie, Indiana University, Bloomington, IN) has been previously demonstrated by multiple lines of evidence including western blot analysis, preadsorption studies, and testing in knockout mice (for details see Eggan and Lewis, 2007). Although CB1Rs are contained in excitatory synapses in the neocortex (Kawamura et al, 2006; Katona et al, 2006), the antibody used in this study exclusively labels symmetric, inhibitory synapses by electron microscopy in both the monkey DLPFC (Eggan and Lewis, 2007) and rodent hippocampus (Katona et al, 1999; Hajos et al, 2000), probably because the much lower level of CB1Rs in excitatory terminals is below the threshold of detectability (Katona et al, 2006; Eggan and Lewis, 2007). Hence, observed differences between subject groups in CB1R immunoreactivity in this study likely reflect changes in the expression of $\mathrm{CB} 1 \mathrm{R}$ protein specifically in inhibitory neurons and axon terminals. Two immunocytochemistry runs were performed for each of the two subject cohorts, with one section from a given subject pair or triad processed together in each run.

\section{Quantification of CB1R Immunoreactivity Levels}

The intensity of CB1R immunoreactivity (expressed as relative optical density (ROD)) in DLPFC area 46 was assessed using a Microcomputer Imaging Device system (Imaging Research, London, Ontario, Canada), without the knowledge of diagnosis or subject number by random coding of slide-mounted sections as described earlier (Eggan and Lewis, 2007). Slide-mounted sections were illuminated on a microscope (Leitz Diaplan; Wild Leitz $\mathrm{GmbH}$, Wetzlar, Germany), images were captured at a final magnification of $\times 74(4.0 \mu \mathrm{m} /$ pixel resolution $)$ by a video camera and digitized, and ROD values were measured by drawing contours of the full cortical thickness for all locations where the gray matter was cut perpendicular to the pial surface. Blood vessels in the contours were excluded to minimize any potential effects of differences in vascularization across brains on ROD measures. The mean $\left( \pm \mathrm{SD}\right.$ ) area sampled per subject was $37.4(8.9) \mathrm{mm}^{2}$ for normal comparison subjects and 37.7 (16.0) $\mathrm{mm}^{2}$ for schizophrenia subjects in cohort 1 , and $35.7(8.8) \mathrm{mm}^{2}$ for normal comparison subjects, 37.3 (16.1) $\mathrm{mm}^{2}$ for schizophrenia subjects, and $34.3(10.0) \mathrm{mm}^{2}$ for MDD subjects in cohort 2 .

To assess CB1R immunoreactivity levels across cortical layers, ROD values were measured within three $\sim 1$-mmwide traverses per section (six traverses per subject) extending from the pial surface to the white matter. The data were divided into 50 bins using Matlab software (The MathWorks, Natick, MA), and the ROD in each layer was determined by dividing the total cortical thickness from the pial surface to white matter into zones of 1-10,10-30, $30-50,50-60,60-80$, and $80-100 \%$, which approximate the locations of layers 1, 2-superficial 3 (3s), deep 3 (3d), 4,5 , and 6, respectively (Pierri et al, 1999). As the highest density of CB1R-immunoreactive (IR) axons occurs in layer 4 and precisely marks the cytoarchitectonic boundaries between layers 3-4 and 4-5 (Eggan and Lewis, 2007), the bins were aligned so that the peak ROD value of each traverse corresponded to the zone representing the middle of layer 4 for every traverse.

All images for slides processed in an experimental run were acquired in the same session under identical room and microscope illuminations and with the same gain and black levels and flatfield correction. All cortical and laminar gray matter measures were corrected by subtracting background ROD values obtained from the white matter of each subject.

\section{Haloperidol-Exposed Monkeys}

To further evaluate the effects of long-term antipsychotic medication exposure on CB1R immunoreactivity levels (Eggan et al, 2008), we studied four male macaque monkeys (Macaca fascicularis) who had received the antipsychotic, haloperidol decanoate, for 9-12 months and four control animals matched for sex, age, and weight (Akil et al, 1999). The mean $( \pm S D)$ dose of haloperidol decanoate was 16.0 (2.1) $\mathrm{mg} / \mathrm{kg}$, was administered by injection every 4 weeks, and yielded trough serum haloperidol concentrations of 4.3 (1.1) ng/ml. Similar concentrations have been associated with a therapeutic response in humans (Volavka et al, 1992), and resulted in extrapyramidal symptoms that were effectively controlled with maintenance administration of benztropine mesylate in all treated animals.

Animals were euthanized in pairs and tissue was processed as described earlier (Dorph-Petersen et al, 2005; Hashimoto et al, 2008a; Sweet et al, 2009). Three DLPFC tissue sections from each animal, separated by $800 \mu \mathrm{m}$, were processed for CB1R immunoreactivity, as described above, except that the anti-CB1R-L15 antibody was diluted $1: 5000$. Levels of CB1R immunoreactivity were measured without the knowledge of animal or drug condition by random coding of slides. CB1R immunoreactivity levels were quantified from images of the ventral bank of the principal sulcus in DLPFC area 46, as described above.

All monkeys were drug naive before onset of haloperidol exposure. All procedures were conducted in accordance with NIH guidelines and were approved by the University of Pittsburgh's Institutional Animal Care and Use Committee.

\section{Statistical Analyses}

To test the effect of diagnosis on CB1R immunoreactivity measures, two analysis of covariance (ANCOVA) models 
were performed using background corrected mean ROD values (Eggan et al, 2008). For cohort 1, the first ANCOVA model used a paired design with ROD entered as the dependent variable, diagnostic group as the main effect, subject pair as a blocking factor, and tissue storage time as a covariate. Subject pairing reduces biological variance by balancing the diagnostic groups for age, sex, and PMI and minimizes inter-assay experimental variance through the parallel processing of tissue samples from each subject pair. However, because subject pairing does not represent a true statistical paired design, a second ANCOVA model was performed using an unpaired design with ROD entered as the dependent variable, diagnostic group as the main effect, and age, sex, PMI, and storage time as covariates. For cohort 2, the first model used a paired design, as described above, to compare each combination of diagnostic groups. The second model used an unpaired design with the same covariates described above, and with a post hoc analysis (least significant difference) to test the between group differences. Sex, age, and PMI did not have a significant effect in either cohort 1 (all $\left.\mathrm{F}_{(1,18)}<0.21 ; p>0.656\right)$ or cohort 2 (all $\left.\mathrm{F}_{(2,35)}<2.4 ; p>0.134\right)$. Storage time also did not have a significant effect in cohort 2 (paired: all $\mathrm{F}_{(1,12)}>3.2 ; p>0.092$; unpaired: $\left.\mathrm{F}_{(1,35)}=0.06 ; p=0.808\right)$ or in the unpaired model for cohort $1 \quad\left(\mathrm{~F}_{(1,18)}=0.21\right.$; $p=0.651)$, but was observed to have a significant effect in the paired model for cohort $1\left(\mathrm{~F}_{(1,10)}=9.12 ; p=0.013\right)$. Regression analysis revealed no significant correlation between storage time and CB1R immunoreactivity levels in normal comparison $(r=0.06 ; p=0.851)$ or schizophrenia $(r=0.42 ; p=0.170)$ subjects, or across all subjects $(r=0.12$; $p=0.579)$.

For laminar density measures, a multivariate analysis of variance (MANOVA) was performed, with ROD for each layer entered as the dependent variable, diagnosis as the main effect, and sex, age, PMI, and storage time as covariates. For all significant values, the least significant difference post hoc test (with $\alpha=0.05$ ) was used to assess the differences between subject groups within each layer.

The effect of potential confounding factors on CB1R immunoreactivity levels across all subjects with schizophrenia (both cohorts 1 and 2) or MDD were evaluated using an ANCOVA model, with ROD entered as the dependent variable, each confounding factor as the main effect, and sex, age, PMI, and storage time as covariates. As tissue from each cohort was processed in a different immunocytochemical experiment, immunocytochemical run was entered as a blocking factor; however, immunocytochemical run was never observed to have a significant effect and was excluded in the reported analyses. As the addition of covariates reduces the degrees of freedom and may diminish the ability to detect small differences, we also evaluated the effect of each potential confounding factor using a simple analysis of variance model (ANOVA).

\section{RESULTS}

\section{Comparison of CB1R Immunoreactivity Levels in Areas} 46 and 9 in Cohort 1

Qualitative examination of tissue sections (Figure 1a) revealed an intensity and pattern of CB1R immunoreactivity in DLPFC area 46 identical to that previously reported in human and monkey area 46 (see Figures 1 and 12A in Eggan and Lewis, 2007) and very similar to that in human area 9 (see Figure 5A in Eggan et al, 2008). Specifically, intense CB1R immunoreactivity was primarily observed in axons and axon varicosities; the density of these structures progressively increased across cortical layers 2 and 3, formed a distinct, dense band in layer 4, fell strikingly in layer 5, and intensified again in layer 6 (Figure 1a). As reported earlier, CB1R immunoreactivity was detectable in only a few cell bodies (Eggan and Lewis, 2007).

In cohort 1 , mean $( \pm S D)$ ROD levels of CB1R immunoreactivity in area 46 were significantly (unpaired: $\mathrm{F}_{(1,18)}=6.3 ; p=0.022 ;$ paired: $\left.\mathrm{F}_{(1,10)}=17.5 ; \quad p=0.002\right)$ $19.1 \%$ lower in schizophrenia subjects $(0.156 \pm 0.023)$ relative to matched normal comparison subjects $(0.193 \pm 0.039)$ (Figure 2a; Supplementary Figure S1), with CB1R immunoreactivity levels lower in the subject with schizophrenia in 9 of 12 pairs (Supplementary Figure S1).

In area 9 from these subjects, CB1R immunoreactivity levels were also significantly $13.9 \%$ lower in subjects with schizophrenia relative to normal comparison subjects (Eggan et al, 2008). Significant positive correlations were present between areas 9 and 46 for CB1R immunoreactivity across all subjects $(r=0.73 ; p<0.001$; Figure $2 \mathrm{~b})$ and for the within-subject pair percent differences in CB1R immunoreactivity $(r=0.62, p=0.032$; Figure $2 c)$.

\section{CB1R Immunoreactivity Levels in Area 46 of Cohort 2}

In cohort 2, the overall density of CB1R-IR axons in area 46 appeared to be lower in schizophrenia subjects relative to both matched normal comparison and matched MDD subjects (Figure 1). Quantitative assessments revealed a significant main effect of diagnosis (unpaired: $\mathrm{F}_{(2,35)}=3.7$; $p=0.036$ ) on CB1R immunoreactivity levels (Figure 3). Post hoc analysis demonstrated that mean levels of CB1R immunoreactivity in schizophrenia subjects $(0.172 \pm 0.043)$ were significantly $20.0 \%$ lower than in normal comparison subjects $(0.215 \pm 0.057 ; p=0.027)$ and significantly $23.3 \%$ lower than in MDD subjects $(0.224 \pm 0.031 ; p=0.022)$, but did not differ between MDD and normal comparison subjects ( $p=0.747$; Figure 3$)$. Paired analyses also demonstrated that CB1R immunoreactivity levels in area 46 were significantly lower in schizophrenia subjects relative to both matched normal comparison (paired: $\mathrm{F}_{(1,12)}=5.4$, $p=0.039$; Supplementary Figure S2A) and matched MDD (paired: $\mathrm{F}_{(1,12)}=16.9 ; p=0.001$; Supplementary Figure S2B) subjects and that the schizophrenia subject had lower CB1R immunoreactivity levels relative to the matched normal comparison subject in 9 of the pairs and relative to the matched MDD subject in 13 of the pairs (Supplementary Figure S2A and B). In contrast, CB1R immunoreactivity levels did not significantly differ between MDD and normal comparison subjects (paired: $\mathrm{F}_{(1,12)}=0.3, p=0.596$; Supplementary Figure S2C).

\section{Laminar Assessment of CB1R Immunoreactivity Levels in Area 46 of Cohort 2}

The distinctive laminar pattern of CB1R immunoreactivity in area 46 was conserved across subject groups (Figure 1). 
a

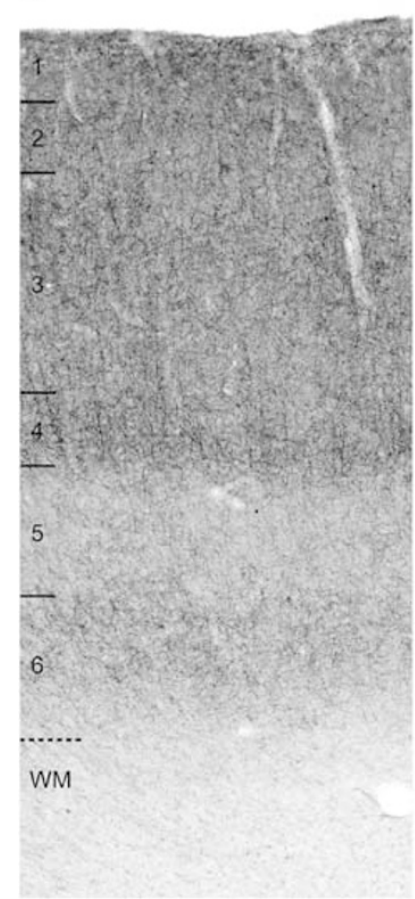

b

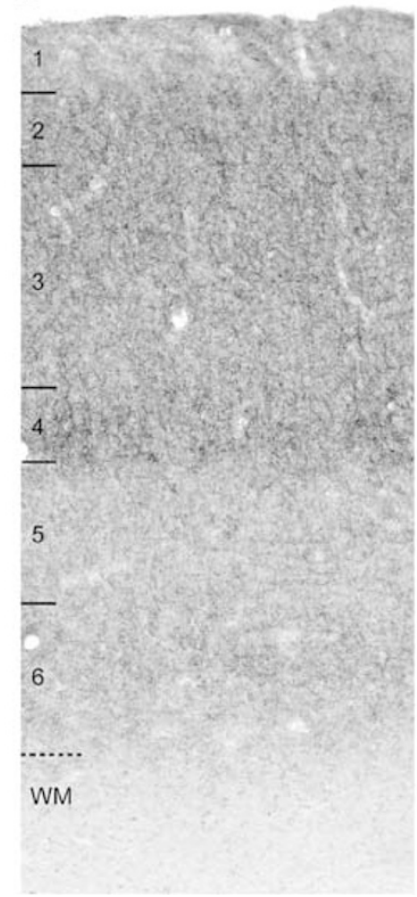

C

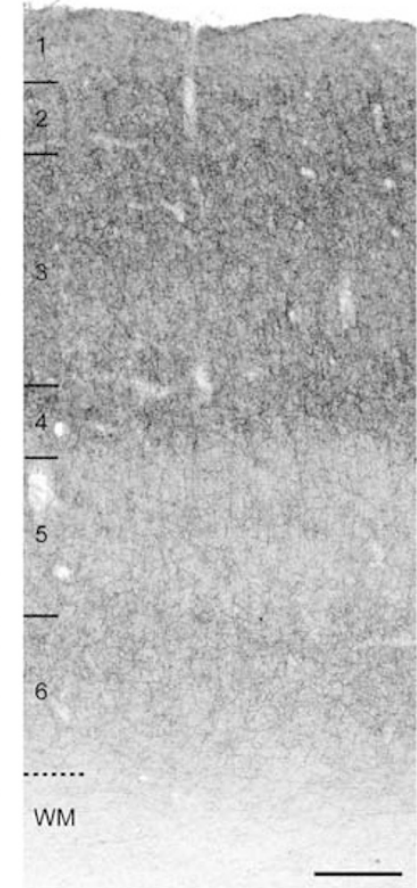

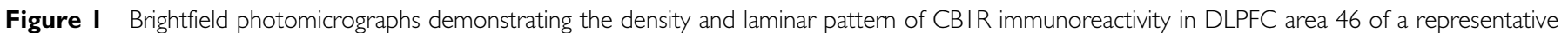

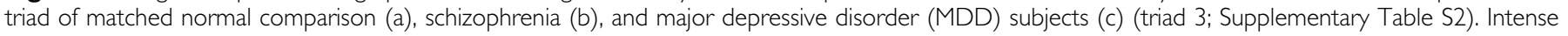

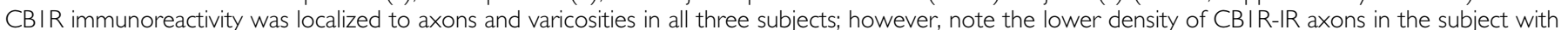

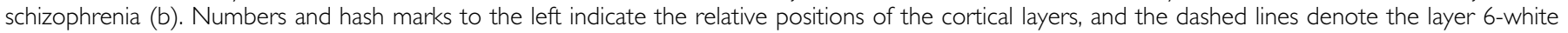
matter (WM) border. Scale bar in (c) $=300 \mu \mathrm{m}$ and applies to all panels.

The levels of CB1R immunoreactivity for the schizophrenia group were lower across all layers relative to both the normal comparison and MDD subject groups, but these differences achieved statistical significance only in layers $1-4\left(\right.$ all $\mathrm{F}_{(2,35)}>3.6$; all $p<0.036$; Figure 4$)$. Post hoc analysis revealed that the schizophrenia group had significantly lower CB1R immunoreactivity in layers $1-4$ (all $p<0.037$ ) relative to the normal comparison group and in layers $1-4$ and layer 6 (all $p<0.039$ ) relative to the MDD group (Figure 4).

\section{Confounding Factors and CB1R Immunoreactivity Levels}

To assess the effect of potential confounding factors on CB1R protein levels, we compared the levels of CB1R immunoreactivity in area 46 from all 26 subjects with schizophrenia with or without a given confound. ANCOVA analysis demonstrated that mean CB1R immunoreactivity levels in the schizophrenia subjects did not differ as a function sex; diagnosis of schizoaffective disorder; suicide; antidepressant, benzodiazepine, or antipsychotic use at the time of death; diagnosis of substance abuse or dependence at the time of death, or history of cannabis use or abuse (all $\mathrm{F} \leqslant 2.8$; all $p \geqslant 0.115$; Figure 5 ). ANOVA analysis also failed to reveal an effect of each potential confounding variable on $\mathrm{CB} 1 \mathrm{R}$ immunoreactivity levels (all $\mathrm{F} \leqslant 1.5$; all $p \geqslant 0.228$ ).

To assess the effect of psychosis or suicide, independent of a diagnosis of schizophrenia, we compared the levels of CB1R immunoreactivity in MDD subjects with or without those factors. The mean levels of CB1R immunoreactivity did not significantly differ in MDD subjects with or without psychotic features (unpaired ANCOVA: $\mathrm{F}_{(1,8)}=2.8 ; p=0.131$; unpaired ANOVA: $\mathrm{F}_{(1,12)}=0.7 ; p=0.419 ;$ Supplementary Figure S3A) or who died by suicide or by other means (unpaired ANCOVA: $\mathrm{F}_{(1,8)}=0.8 ; p=0.403$; unpaired ANOVA: $\mathrm{F}_{(1,12)}=0.6 ; p=0.468$; Supplementary Figure $\left.\mathrm{S} 3 \mathrm{~B}\right)$.

\section{CB1R Immunoreactivity Levels in Haloperidol-Exposed Monkeys}

To assess the effect of antipsychotic medications on CB1R protein levels, we compared CB1R immunoreactivity levels in area 46 from monkeys chronically exposed to haloperidol or vehicle. Mean $( \pm S D)$ CB1R immunoreactivity levels in area 46 were $11.5 \%$ higher (unpaired: $\mathrm{F}_{(1,7)}=3.9 ; p=0.095$; paired: $\left.\mathrm{F}_{(1,3)}=10.8 ; p=0.046\right)$ in haloperidol-exposed monkeys $(0.188 \pm 0.007)$ relative to matched control monkeys (0.168 \pm 0.018 ; Figure 6$)$. CB1R immunoreactivity levels were higher in the haloperidol-exposed monkey in each of the four pairs (Figure 6).

\section{DISCUSSION}

We recently reported that $\mathrm{CB} 1 \mathrm{R}$ mRNA and protein levels were significantly lower in DLPFC area 9 in a cohort of 23 matched pairs of schizophrenia and normal comparison subjects (Eggan et al, 2008). In this study, we found that the levels of CB1R immunoreactivity (1) are similarly reduced 
a
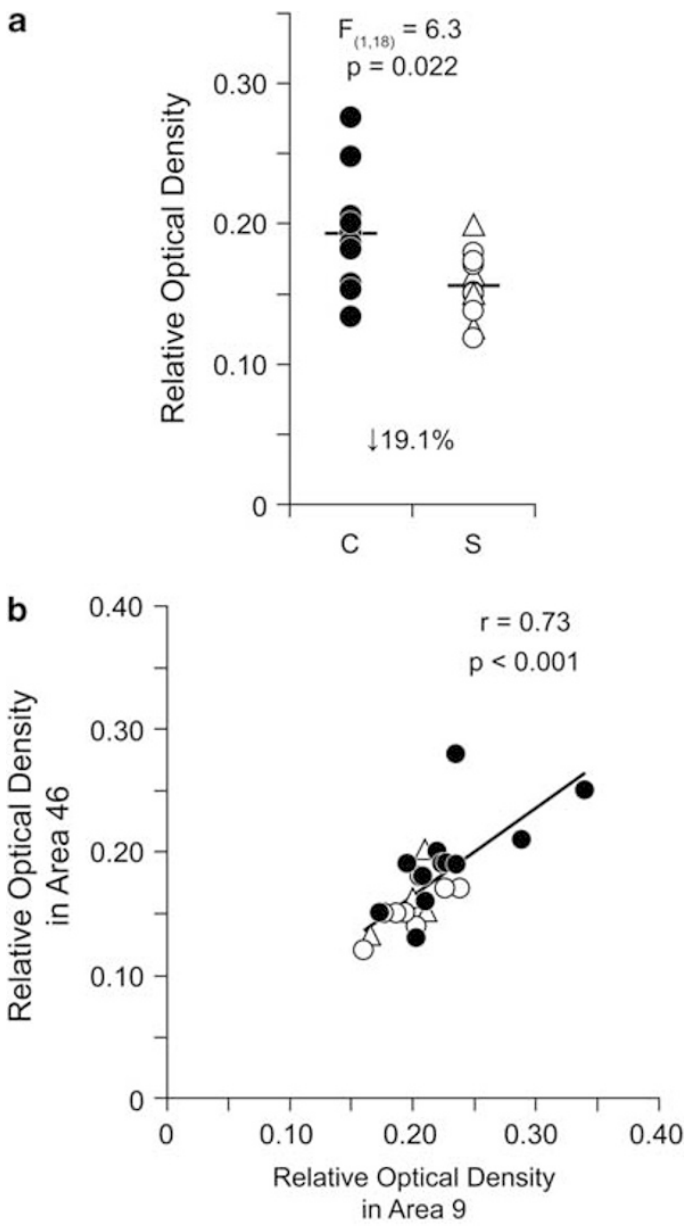

C

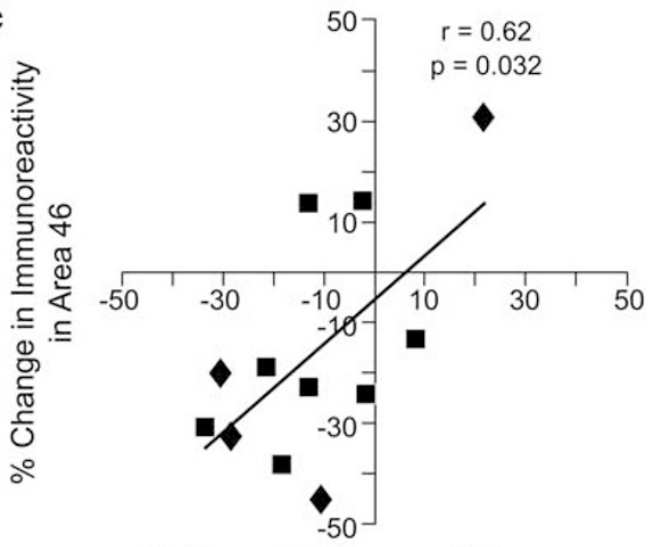

$\%$ Change in Immunoreactivity in Area 9

Figure 2 Lower CBIR immunoreactivity in DLPFC area 46 of subjects with schizophrenia from cohort I. (a) Unpaired analysis of mean cortical ROD levels of CBIR immunoreactivity in normal comparison subjects (C; filled circles) and matched subjects with schizophrenia (S; open circles) or schizoaffective disorder (open triangles). Mean values for each subject group are indicated by horizontal bars. The ROD levels of CBIR immunoreactivity (b) and within-pair percent change in CBIR immunoreactivity levels (c) measured in area 46 correlated with those previously reported in area 9 (Eggan et al, 2008) of the same subjects with schizophrenia. (b) Closed circles denote normal comparison subjects and open circles or triangles denote schizophrenia or schizoaffective disorder subjects, respectively. (c) Closed squares denote matched normal comparison and schizophrenia subject pairs and closed diamonds denote normal comparison and schizoaffective disorder subject pairs.

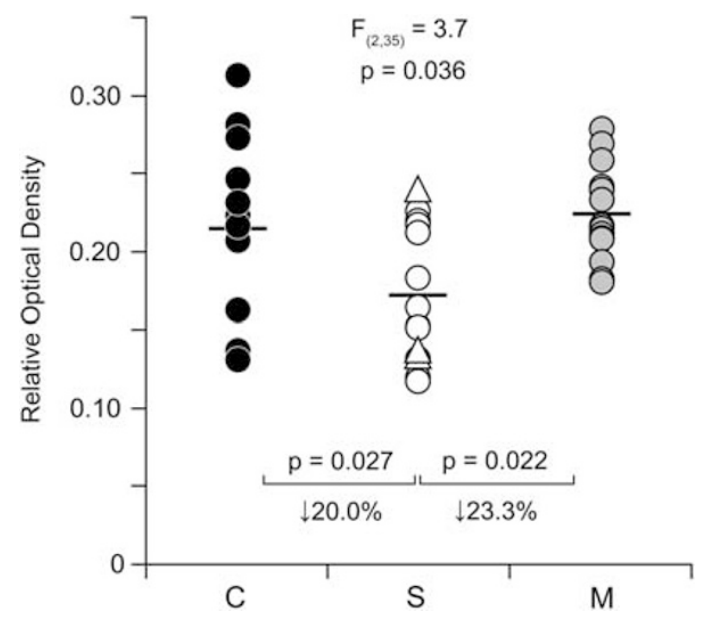

Figure 3 Lower CBIR immunoreactivity in DLPFC area 46 of subjects with schizophrenia from cohort 2. Unpaired analysis of cortical ROD levels of CBIR immunoreactivity in matched normal comparison subjects (C; closed circles), subjects with schizophrenia (S; open circles) or schizoaffective disorder (open triangles), and subjects with major depressive disorder (M; gray circles). Mean values for each subject group are indicated by horizontal bars.

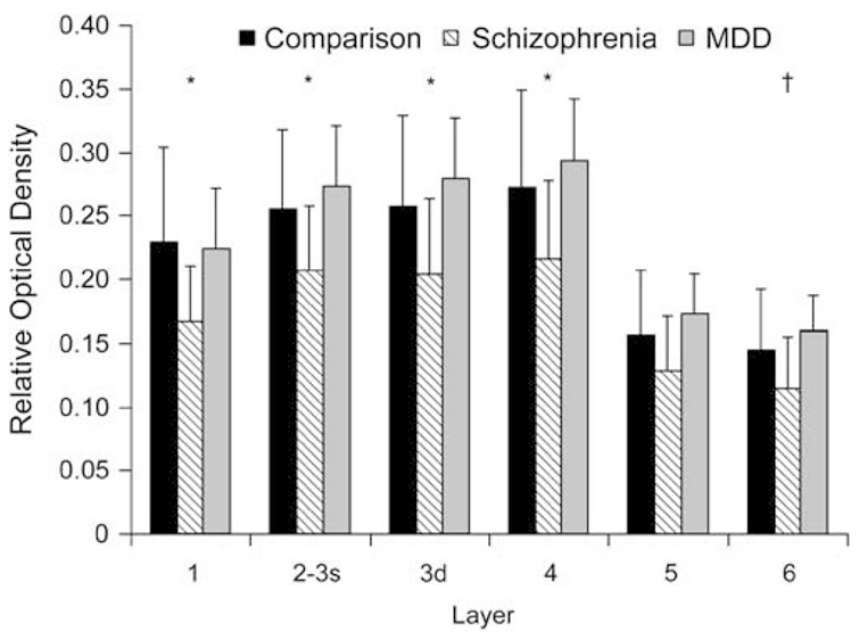

Figure 4 Lower CBIR immunoreactivity levels across cortical layers in DLPFC area 46 of subjects from cohort 2. The distinctive laminar pattern of CBIR immunoreactivity was conserved across subject groups; however, the levels of CBIR immunoreactivity for the schizophrenia group were reduced across all layers relative to the normal comparison and MDD groups. Comparison of mean $( \pm S D)$ ROD levels of CBIR immunoreactivity in each cortical layer revealed that $C B I R$ immunoreactivity levels were significantly $(p<0.05)$ lower in schizophrenia subjects in layers $1-4$ relative to both normal comparison and MDD subjects (*), and in layer 6 relative to MDD subjects $(\dagger)$. 3s indicates superficial layer $3 ; 3$ indicates deep layer 3.

in areas 9 and 46 of the same subjects with schizophrenia; (2) are also reduced in area 46 in a new cohort of schizophrenia subjects; (3) are not altered in subjects with MDD, with or without the presence of psychotic features or death by suicide; and (4) are not apparently influenced by antipsychotic exposure or other confounding factors. These data suggest that the lower levels of CB1R protein may be common and conserved across DLPFC regions in 


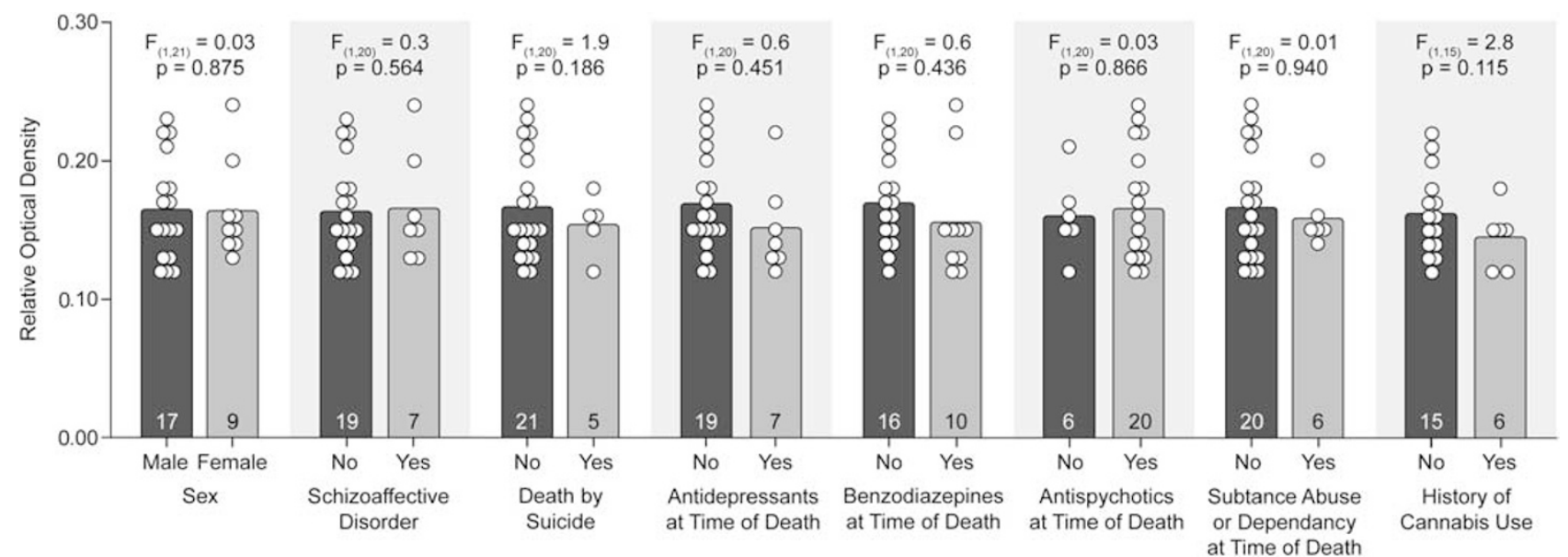

Figure 5 The effects of confounding factors on CBIR immunoreactivity levels in schizophrenia. Mean (bar) and individual subject (circles) ROD levels of CBIR immunoreactivity in the subjects with schizophrenia grouped by potential confounding factors. Neither sex, diagnosis of schizoaffective disorder, suicide, antidepressant medication use at the time of death, benzodiazepine use at the time of death, antipsychotic medication use at the time of death, diagnosis of substance abuse/dependence at the time of death, nor history of cannabis use/abuse significantly affected levels of CBIR immunoreactivity. Numbers in bars indicate the number of subjects with schizophrenia in each category. A history of cannabis use was unknown for five of the schizophrenia subjects in cohort 2; thus those subjects were not included in the analysis.

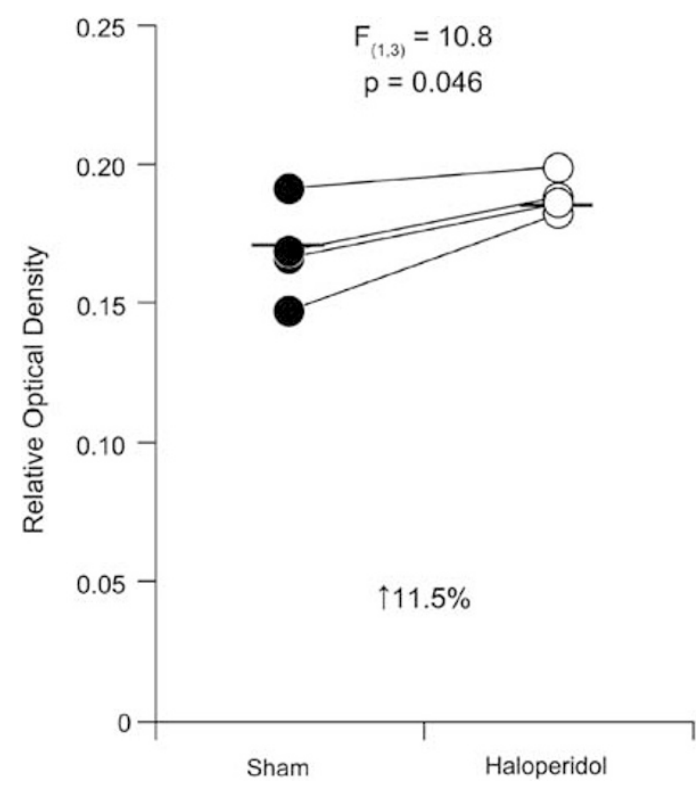

Figure 6 Higher CBIR immunoreactivity in DLPFC area 46 of monkeys chronically exposed to the antipsychotic haloperidol. Paired analysis of mean cortical ROD levels of CBIR immunoreactivity in sham-exposed monkeys (filled circles) and matched monkeys chronically exposed to haloperidol (open circles). Mean values for each subject group are indicated by horizontal bars.

schizophrenia, and show at least some specificity for the disease process of this illness.

\section{Specificity of Lower CB1R Levels to the Disease Process of Schizophrenia}

Convergent lines of evidence suggest that lower CB1R protein levels in the DLPFC of subjects with schizophrenia are not due to comorbid depression or suicide, and are not a consequence of psychosis in general. First, in contrast to schizophrenia, no alterations in CB1R immunoreactivity levels were observed in MDD subjects. In another study, CB1R protein levels in MDD subjects, assessed by western blot analysis, were reported to be significantly increased in area 9 of MDD subjects (Hungund et al, 2004). Preclinical data demonstrate that CB1R agonists and endocannabinoid enhancers possess antidepressant-like properties (Bortolato et al, 2007; Mangieri and Piomelli, 2007) and clinical evidence suggests that the $\mathrm{CB} 1 \mathrm{R}$ antagonist rimonabant increases the risk of depression and suicidality (Christensen et al, 2007). Thus, although this interpretation is speculative, higher CB1R protein levels in MDD subjects might represent a compensatory, albeit insufficient, response to ameliorate depressive symptoms. Second, the lower levels of CB1R immunoreactivity in schizophrenia were not associated with death by suicide and CB1R immunoreactivity levels did not differ between MDD subjects with or without death by suicide. Third, CB1R immunoreactivity levels were not significantly affected by the presence of psychotic features in some of the MDD subjects. Thus, the lower levels of CB1R protein observed in schizophrenia do not seem to be attributable to depression, suicide, or psychosis, and thus might be specific to the disease process of schizophrenia.

This interpretation is further supported by the finding that lower levels of CB1R immunoreactivity in DLPFC area 46 of subjects with schizophrenia cannot be attributed to pharmacological treatment of the illness; CB1R immunoreactivity levels in schizophrenia subjects did not differ as a function of benzodiazepine, antidepressant, or antipsychotic medication use at the time of death. A recent study reported that CB1R protein levels, assessed by western blot analysis, were significantly lower in the prefrontal cortex of antipsychotic-treated, but not of drug-free, schizophrenia subjects (Uriguen et al, 2009); however, several other lines of evidence indicate that prefrontal CB1R levels are not affected by antipsychotic medications. First, CB1R mRNA expression levels were unaltered in the DLPFC of monkeys 
chronically exposed to therapeutic serum levels of either typical (haloperidol) or atypical (olanzapine) antipsychotic medications (Eggan et al, 2008). Similarly, exposure to a variety of antipsychotic medications did not alter the density of CB1Rs, measured by radioligand binding, in the rat prefrontal cortex (Sundram et al, 2005; Wiley et al, 2008). Consistent with these observations, the six subjects with schizophrenia in this study (three subjects each from cohorts 1 and 2; Supplementary Tables S1 and S2) who were off antipsychotic medications at the time death all had lower CB1R immunoreactivity levels in area 46 than their matched normal comparison subjects. Second, the mean withinsubject pair percent difference in CB1R immunoreactivity was larger in those schizophrenia subjects who were not receiving antipsychotic medications at the time of death $(-33.3 \% ; n=6)$ compared with those who were receiving antipsychotic medications at the time of death $(-14.9 \%$; $n=20$ ). Third, although the sample size was small, CB1R immunoreactivity levels showed a trend to being higher in area 46 from monkeys after long-term exposure to haloperidol at doses that mimicked the therapeutic treatment of schizophrenia (Figure 6). Together, these data suggest that exposure to antipsychotic medications does not account for the lower CB1R immunoreactivity levels observed in schizophrenia, and might have actually blunted the disease-related decrease.

The lower levels of CB1R immunoreactivity also do not seem to be driven by other potential confounding factors that are commonly associated with schizophrenia. For example, consistent with our earlier findings for CB1R mRNA (by in situ hybridization) and protein (by radioimmunocytochemistry) in area 9 (Eggan et al, 2008), CB1R immunoreactivity levels in area 46 of schizophrenia subjects did not differ as a function of sex, a diagnosis of schizoaffective disorder, or a diagnosis of substance abuse/dependence at the time of death (Figure 5).

Although our analysis considered all substances of abuse together (except cannabis that was assessed separately), nicotine and alcohol exhibit distinct interactions with cannabinoid signaling; thus, use of these substances by schizophrenia subjects may represent potential confounds. For example, evidence suggests a function for CB1Rs in modulating nicotine addiction (Fagerstrom and Balfour, 2006) and the high prevalence and extensive use of nicotine by individuals with schizophrenia is well documented. The effect of nicotine is difficult to control for in postmortem studies since, even if schizophrenia and comparison subjects could be matched for pack years of smoking history, individuals with schizophrenia are likely to have had greater nicotine exposure due to their tendency to extract more nicotine per cigarette. However, to our knowledge, only one study has investigated the effect of nicotine exposure on CB1R protein levels (Marco et al, 2007). This study in rodents showed that subchronic exposure to nicotine during adolescence produced a significant increase in CB1R immunoreactivity in the hippocampus measured by western blot analysis. Thus, a history of nicotine use in our schizophrenia subjects may have actually blunted the observed reduction in CB1R protein levels. In rodents, chronic ethanol exposure may downregulate CB1Rs in some brain regions (Basavarajappa and Hungund, 2002; Hungund and Basavarajappa, 2004); however, region-specific effects of drugs on CB1R expression are reported (Sundram et al, 2005; Wiley et al, 2008). Indeed, CB1R mRNA expression is reported to be increased 2.6-fold in the prefrontal cortex of alcohol-exposed rats (Rimondini et al, 2002). Consistent with these data, in humans, CB1R ligand-binding levels are higher in both alcoholic subjects and alcoholic suicide victims compared with normal control subjects or non-alcoholic suicide victims, respectively (Hungund et al, 2004; Vinod et al, $2005)$. In our study, the mean ( \pm SD) level of CB1R immunoreactivity was $0.158 \pm 0.021$ in the subjects with schizophrenia who had a diagnosis of alcohol abuse/ dependence at the time of death $(n=6) \quad(11.2 \%$ lower relative to matched comparison subjects) compared with $0.166 \pm 0.039(22.1 \%$ lower relative to matched comparison subjects) in the subjects with schizophrenia without a diagnosis of alcohol abuse/dependence $(n=20)$. Furthermore, mean $( \pm S D)$ CB1R immunoreactivity levels did not differ $\left(\mathrm{F}_{(1,20)}=0.006 ; p=0.940\right)$ between those schizophrenia subjects with or without a diagnosis of alcohol abuse/ dependence at the time of death. Thus, a history of alcohol abuse/dependency at the time of death does not seem to account for the observed decreases in CB1R protein in subjects with schizophrenia.

Finally, the findings from this study suggest that cannabis use does not seem to account for lower levels of prefrontal CB1R immunoreactivity in schizophrenia. First, consistent with our earlier findings of CB1R mRNA and protein in area 9 (Eggan et al, 2008), CB1R immunoreactivity levels in area 46 of schizophrenia subjects did not differ as a function of a prior history of cannabis use. Second, in cohort 2 , both the schizophrenia and matched MDD subject were known to have a history of cannabis use for three subject pairs, and for five of the subject pairs both members did not have a history of cannabis use (Supplementary Table S2). In the five subject pairs without a history of cannabis use, the mean level of CB1R immunoreactivity in area 46 was $23.5 \%$ lower in schizophrenia subjects relative to the matched MDD subjects. Similarly, in the three subject pairs with a history of cannabis use, the mean level of CB1R immunoreactivity was $26.9 \%$ lower in schizophrenia subjects relative to the matched MDD subjects. Consistent with these observations, measures of CB1Rs do not seem to be permanently altered in the prefrontal cortex of either monkeys or rodents after exposure to CB1R agonists (Westlake et al, 1991; Rubino et al, 1994; Zhuang et al, 1998; Romero et al, 1998; Garcia-Gil et al, 1999). However, the findings of the effects of CB1R agonist exposure on CB1R levels are mixed and seem to be dependent on the dose administered, length of exposure, and the brain region studied (Romero et al, 1995, 1997; Zhuang et al, 1998; SimSelley, 2003); thus, although an effect of cannabis use cannot be ruled out, the findings are consistent with the idea that earlier cannabis use does not account for lower levels of prefrontal CB1R immunoreactivity in schizophrenia.

In concert, these data suggest that neither depression, suicide, psychosis, antipsychotic medications, other potential confounding factors, nor a prior history of cannabis use seem to account for lower levels of CB1R immunoreactivity in the DLPFC from two independent cohorts of subjects with schizophrenia. Thus, although our analysis of potential confounding factors may suffer from type II error, the 
findings all converge on the hypothesis that lower CB1R levels in the DLPFC may reflect the disease process of schizophrenia.

In contrast, CB1R-IR structures in the anterior cingulate cortex were reported to be unaltered in schizophrenia subjects (Koethe et al, 2007); importantly, the specificity of the antibody used was not adequately tested and the reported anatomical localization and laminar distribution of CB1R immunoreactivity was inconsistent with those previously demonstrated in rodent, monkey, and human cortex in studies using well-characterized CB1R antibodies (Katona et al, 2000; Egertová and Elphick, 2000; Morozov and Freund, 2003; Bodor et al, 2005; Eggan and Lewis, 2007; Eggan et al, 2008). In addition, increased binding of the CB1R agonist $\left[{ }^{3} \mathrm{H}\right] \mathrm{CP}-55940$ was reported in the DLPFC (Dean et al, 2001) and posterior cingulate cortex (Newell et al, 2006) of schizophrenia subjects. However, increased $\left[{ }^{3} \mathrm{H}\right] \mathrm{CP}-55940$ binding might reflect differences other than the amount of receptor present because CB1Rs contain an allosteric modulation site that, when bound, elicits a conformational change in the receptor increasing the affinity of $\left[{ }^{3} \mathrm{H}\right] \mathrm{CP}-55940$ for the orthosteric-binding site (Price et al, 2005; Horswill et al, 2007). Increased binding of the CB1R antagonist $\left[{ }^{3} \mathrm{H}\right] \mathrm{SR} 141716$ was also reported in the anterior cingulate cortex of subjects with schizophrenia (Zavitsanou et al, 2004); however, SR141716 has functional effects in CB1R knockout mice, suggesting that it binds receptors other than the CB1R (Breivogel et al, 2001; Hajos et al, 2001). Importantly, saturation and competition experiments were not performed in any of these studies to determine whether the number of binding sites or the affinity for the ligand were altered in the illness. Thus, the binding of these radioligands may not represent the relative amount of CB1R protein present. However, it is possible that schizophrenia is associated with higher levels of cellular membrane-bound CB1Rs with overall CB1R mRNA and protein levels downregulated due to reduced internalization of CB1Rs. Studies of CB1R binding with specific ligands, and protein and mRNA levels in adjacent tissue sections are necessary to address this possibility; the advent of new CB1R-specific PET ligands (Horti et al, 2006; Burns et al, 2007; Yasuno et al, 2008; Terry et al, 2010) could be useful in this regard, as well as allowing for the assessment of CB1R levels in vivo.

\section{Pathophysiological Significance of Lower CB1R Protein Levels in Schizophrenia}

Although CB1R protein is present in both inhibitory and excitatory cortical axon terminals (Bodor et al, 2005; Kawamura et al, 2006; Katona et al, 2006; Eggan and Lewis, 2007), lower CB1R immunoreactivity levels in schizophrenia likely represent a reduced amount of CB1R protein specifically in the axons and terminals of CCK-containing GABA neurons because (1) CCK-containing axon terminals contain much higher levels of CB1Rs and are more sensitive to the effects of CB1R agonists than are pyramidal cell axon terminals (Marsicano and Lutz, 1999; Ohno-Shosaku et al, 2002); (2) CB1R- and CCK-containing structures in macaque monkey DLPFC exhibit similar overall distribution patterns and undergo nearly identical changes in laminar distribution during postnatal development (Oeth and Lewis, 1993; Eggan et al, 2010); and (3) the antibody used in this study exclusively labels symmetric, inhibitory synapses (Katona et al, 1999, 2001; Eggan and Lewis, 2007). Furthermore, alterations in CB1R mRNA levels in area 9 of schizophrenia subjects strongly correlate with those of $\mathrm{GAD}_{67}$ and CCK mRNAs, suggesting that lower CB1R protein levels in the DLPFC in schizophrenia accompany deficient GABA synthesis in CCK neurons (Eggan et al, 2008).

The lower levels of CB1R protein in the DLPFC of schizophrenia subjects could be secondary to increased endocannabinoid levels. Indeed, elevated levels of the endocannabinoid anandamide have been reported in the blood and cerebral spinal fluid of schizophrenia patients (Leweke et al, 1999; De Marchi et al, 2003; Giuffrida et al, 2004). However, the principal endocannabinoid for CB1Rs in the cortex and hippocampus seems to be 2-arachidonoylglycerol (2-AG), not anandamide (Kim and Alger, 2004; Makara et al, 2005; Hashimotodani et al, 2007); however, 2-AG levels cannot be reliably assessed in postmortem tissue and whether 2-AG levels are elevated in the DLPFC of schizophrenia subjects is unknown.

Alternatively, the lower levels of CB1R immunoreactivity in schizophrenia could represent a compensatory downregulation of $\mathrm{CB} 1 \mathrm{R}$ protein in response to deficient GABA synthesis in the DLPFC (Eggan et al, 2008). Reduced $\mathrm{GAD}_{67}$ mRNA levels in the DLPFC are one of the most consistently replicated findings in schizophrenia and are thought to contribute, at least in part, to DLPFC-related working memory deficits in the illness (Lewis et al, 2005; Akbarian and Huang, 2006). In the DLPFC, normal GABA-mediated neurotransmission is necessary for working memory function (Sawaguchi et al, 1988; Rao et al, 2000). In particular, networks of interconnected GABA interneurons are essential for the synchronization of large ensembles of neurons (Connors and Long, 2004), including $\gamma$-band oscillations that increase in the DLPFC with working memory load in humans (Howard et al, 2003). As the activation of CB1Rs suppress GABA neurotransmission in the DLPFC, a lower density of CB1Rs could, by reducing the endocannabinoid-mediated block of GABA release from the terminals of CB1R/CCK-containing interneurons, compensate for lower levels of $\mathrm{GAD}_{67}$-mediated GABA synthesis in those neurons and contribute to a partial, albeit insufficient, normalization of $\gamma$-band power and working memory function.

This interpretation is supported by the finding that, in contrast to the common and conserved lower levels of CB1R immunoreactivity in the DLPFC of schizophrenia subjects, no alterations in CB1R immunoreactivity levels were observed in the DLPFC of MDD subjects in this study. Importantly, other studies report that $\mathrm{GAD}_{67}$ mRNA expression levels are unaltered in the DLPFC of MDD subjects (Guidotti et al, 2000; Morris and Lewis, 2009). These data suggest that working memory impairments in MDD and schizophrenia may arise from disparate mechanisms. Consistent with this idea, a recent longitudinal study demonstrated that children who developed adult schizophrenia exhibited premorbid developmental impairments including static deficits in cognitive functions such as reasoning and conceptualization and developmental lags in other cognitive functions such as attention and working 
memory, whereas children who later developed recurrent depression did not exhibit those premorbid cognitive patterns (Reichenberg et al, 2010).

If $\mathrm{CB} 1 \mathrm{R}$ downregulation is compensatory for deficient GABA neurotransmission, then reduced levels of CB1R protein may not be restricted to the DLPFC, but may also occur in other cortical regions that exhibit deficient GABA neurotransmission in schizophrenia. For example, overall $\mathrm{GAD}_{67}$ mRNA levels, and the magnitude of $\mathrm{GAD}_{67} \mathrm{mRNA}$ alterations in schizophrenia, are similar across DLPFC area 9, and anterior cingulate, primary motor, and primary visual cortices, suggesting that impaired GABA neurotransmission may broadly contribute to cortical dysfunction in schizophrenia (Hashimoto et al, 2008b). However, CB1R immunoreactivity levels are heterogeneous across cortical regions, with regions associated with higher cognitive functions, such as the DLPFC, containing much higher densities of CB1R-containing axons than primary motor or visual cortices (Eggan and Lewis, 2007). These data suggest that the capacity for endocannabinoid-mediated compensatory responses to GABA deficits may differ across cortical regions, with lower levels of $\mathrm{CB} 1 \mathrm{R}$ protein being a particularly salient compensation for deficient GABA neurotransmission in the DLPFC.

\section{ACKNOWLEDGEMENTS}

This work was supported by an NARSAD Distinguished Investigator Award and by National Institutes of Health grants $\mathrm{MH}-043784$ and $\mathrm{MH}-084053$. We gratefully acknowledge the efforts of the research staff of the Translational Neuroscience Program and thank the members of the Clinical Services and Diagnostics Core of the Conte Center for the Neuroscience of Mental Disorders (MH-084053) for their assistance in diagnostic assessments. We thank Dr Ken Mackie for kindly donating the CB1R antibody, Mary Brady for assistance with the graphics, and Jim Kosakowski for Matlab program development.

\section{DISCLOSURE}

David A Lewis currently receives investigator-initiated research support from the BMS Foundation, Bristol-Myers Squibb, Curridium, and Pfizer, and in 2007-2009 served as a consultant in the areas of target identification and validation and new compound development to AstraZeneca, BioLine RX, Bristol-Myers Squibb, Hoffman-Roche, Lilly, Merck, Neurogen, and SK Life Science. All other authors declare that, except for income received from their primary employer, no financial support or compensation has been received from any individual or corporate entity over the past 3 years for research or professional service, and there are no personal financial holdings that could be perceived as constituting a potential conflict of interest.

\section{REFERENCES}

Akbarian S, Huang HS (2006). Molecular and cellular mechanisms of altered GAD1/GAD67 expression in schizophrenia and related disorders. Brain Res Rev 52: 293-304.
Akil M, Pierri JN, Whitehead RE, Edgar CL, Mohila C, Sampson AR et al (1999). Lamina-specific alterations in the dopamine innervation of the prefrontal cortex in schizophrenic subjects. Am J Psychiatry 156: 1580-1589.

Ameri A (1999). The effects of cannabinoids on the brain. Prog Neurobiol 58: 315-348.

Basavarajappa BS, Hungund BL (2002). Neuromodulatory role of the endocannabinoid signaling system in alcoholism: an overview. Prostaglandins Leukot Essent Fatty Acids 66: 287-299.

Beneyto M, Sibille E, Lewis DA (2009). Human postmortem brain research in mental illness syndromes. In: Charney DS, Nestler EJ (eds). Neurobiology of Mental Illness. Oxford University Press: New York. pp 202-214.

Bersani G, Orlandi V, Kotzalidis GD, Pancheri P (2002). Cannabis and schizophrenia: impact on onset, course, psychopathology and outcomes. Eur Arch Psychiatry Clin Neurosci 252: 86-92.

Bodor AL, Katona I, Nyiri G, Mackie K, Ledent C, Hajos $\mathrm{N}$ et al (2005). Endocannabinoid signaling in rat somatosensory cortex: laminar differences and involvement of specific interneuron types. J Neurosci 25: 6845-6856.

Bortolato M, Mangieri RA, Fu J, Kim JH, Arguello O, Duranti A et al (2007). Antidepressant-like activity of the fatty acid amide hydrolase inhibitor URB597 in a rat model of chronic mild stress. Biol Psychiatry 62: 1103-1110.

Breivogel CS, Griffin G, Di Marzo V, Martin BR (2001). Evidence for a new $G$ protein-coupled cannabinoid receptor in mouse brain. Mol Pharmacol 60: 155-163.

Buckley PF, Miller BJ, Lehrer DS, Castle DJ (2009). Psychiatric comorbidities and schizophrenia. Schizophr Bull 35: 383-402.

Burns HD, Van Laere K, Sanabria-Bohorquez S, Hamill TG, Bormans G, Eng WS et al (2007). [18F]MK-9470, a positron emission tomography (PET) tracer for in vivo human PET brain imaging of the cannabinoid-1 receptor. Proc Natl Acad Sci U S A 104: 9800-9805.

Christensen R, Kristensen PK, Bartels EM, Blidda H, Astrup A (2007). Efficacy and safety of the weight-loss drug rimonabant: a meta-analysis of randomised trials. Lancet 370: 1706-1713.

Connors BW, Long MA (2004). Electrical synapses in the mammalian brain. Annu Rev Neurosci 27: 393-418.

Cruz DA, Weaver CL, Lovallo EM, Melchitzky DS, Lewis DA (2009). Selective alterations in postsynaptic markers of chandelier cell inputs to cortical pyramidal neurons in subjects with schizophrenia. Neuropsychopharmacology 34: 2112-2124.

D'Souza DC, Abi-Saab WM, Madonick S, Forselius-Bielen K, Doersch A, Braley G et al (2005). Delta-9-tetrahydrocannabinol effects in schizophrenia: implications for cognition, psychosis, and addiction. Biol Psychiatry 57: 594-608.

Daviss SR, Lewis DA (1995). Local circuit neurons of the prefrontal cortex in schizophrenia: selective increase in the density of calbindin-immunoreactive neurons. Psychiatry Res 59: 81-96.

De Marchi N, De Petrocellis L, Orlando P, Daniele F, Fezza F, Di Marzo V (2003). Endocannabinoid signalling in the blood of patients with schizophrenia. Lipids Health Dis 2: 5.

Dean B, Sundram S, Bradbury R, Scarr E, Copolov D (2001). Studies on $[3 \mathrm{H}] \mathrm{CP}-55940$ binding in the human central nervous system: regional specific changes in density of cannabinoid-1 receptors associated with schizophrenia and cannabis use. Neuroscience 103: 9-15.

Deng C, Han M, Huang XF (2007). No changes in densities of cannabinoid receptors in the superior temporal gyrus in schizophrenia. Neurosci Bull 23: 341-347.

Dorph-Petersen K-A, Pierri JN, Perel JM, Sun Z, Sampson AR, Lewis DA (2005). The influence of chronic exposure to antipsychotic medications on brain size before and after tissue fixation: a comparison of haloperidol and olanzapine in macaque monkeys. Neuropsychopharmacology 30: 1649-1661. 
Egertová M, Elphick MR (2000). Localisation of cannabinoid receptors in the rat brain using antibodies to the intracellular Cterminal tail of CB. J Comp Neurol 422: 159-171.

Eggan SM, Hashimoto T, Lewis DA (2008). Reduced cortical cannabinoid 1 receptor messenger RNA and protein expression in schizophrenia. Arch Gen Psychiatry 65: 772-784.

Eggan SM, Lewis DA (2007). Immunocytochemical distribution of the cannabinoid $\mathrm{CB} 1$ receptor in the primate neocortex: a regional and laminar analysis. Cereb Cortex 17: 175-191.

Eggan SM, Mizoguchi Y, Stoyak SR, Lewis DA (2010). Development of cannabinoid 1 receptor protein and messenger RNA in monkey dorsolateral prefrontal cortex. Cereb Cortex 20: 1164-1174.

Elvevag B, Goldberg TE (2000). Cognitive impairment in schizophrenia is the core of the disorder. Crit Rev Neurobiol 14: 1-21.

Fagerstrom K, Balfour DJ (2006). Neuropharmacology and potential efficacy of new treatments for tobacco dependence. Expert Opin Investig Drugs 15: 107-116.

Galarreta M, Erdelyi F, Szabo G, Hestrin S (2004). Electrical coupling among irregular-spiking GABAergic interneurons expressing cannabinoid receptors. J Neurosci 24: 9770-9778.

Garcia-Gil L, Romero J, Ramos JA, Fernandez-Ruiz JJ (1999). Cannabinoid receptor binding and mRNA levels in several brain regions of adult male and female rats perinatally exposed to delta9-tetrahydrocannabinol. Drug Alcohol Depend 55: $127-136$

Giuffrida A, Leweke FM, Gerth CW, Schreiber D, Koethe D, Faulhaber J et al (2004). Cerebrospinal anandamide levels are elevated in acute schizophrenia and are inversely correlated with psychotic symptoms. Neuropsychopharmacology 29: 2108-2114.

Guidotti A, Auta J, Davis JM, Gerevini VD, Dwivedi Y, Grayson DR et al (2000). Decrease in reelin and glutamic acid decarboxylase $_{67}\left(\mathrm{GAD}_{67}\right)$ expression in schizophrenia and bipolar disorder. Arch Gen Psychiatry 57: 1061-1069.

Hajos N, Katona I, Naiem SS, Mackie K, Ledent C, Mody I et al (2000). Cannabinoids inhibit hippocampal GABAergic transmission and network oscillations. Eur J Neurosci 12: 3239-3249.

Hajos N, Ledent C, Freund TF (2001). Novel cannabinoid-sensitive receptor mediates inhibition of glutamatergic synaptic transmission in the hippocampus. Neuroscience 106: 1-4.

Hashimoto T, Arion D, Unger T, Maldonado-Aviles JG, Morris HM, Volk DW et al (2008a). Alterations in GABA-related transcriptome in the dorsolateral prefrontal cortex of subjects with schizophrenia. Mol Psychiatry 13: 147-161.

Hashimoto T, Bazmi HH, Mirnics K, Wu Q, Sampson AR, Lewis DA (2008b). Conserved regional patterns of GABA-related transcript expression in the neocortex of subjects with schizophrenia. Am J Psychiatry 165: 479-489.

Hashimotodani Y, Ohno-Shosaku T, Kano M (2007). Presynaptic monoacylglycerol lipase activity determines basal endocannabinoid tone and terminates retrograde endocannabinoid signaling in the hippocampus. J Neurosci 27: 1211-1219.

Horswill JG, Bali U, Shaaban S, Keily JF, Jeevaratnam P, Babbs AJ et al (2007). PSNCBAM-1, a novel allosteric antagonist at cannabinoid CB1 receptors with hypophagic effects in rats. $\mathrm{Br}$ J Pharmacol 152: 805-814.

Horti AG, Fan H, Kuwabara H, Hilton J, Ravert HT, Holt DP et al (2006). 11C-JHU75528: a radiotracer for PET imaging of CB1 cannabinoid receptors. J Nucl Med 47: 1689-1696.

Howard MW, Rizzuto DS, Caplan JB, Madsen JR, Lisman J, Aschenbrenner-Scheibe $\mathrm{R}$ et al (2003). Gamma oscillations correlate with working memory load in humans. Cereb Cortex 13: $1369-1374$.

Hsu S-M, Raine L, Fanger H (1981). Use of avidin-biotinperoxidase complex $(\mathrm{ABC})$ in immunoperoxidase techniques: a comparison between $\mathrm{ABC}$ and unlabeled antibody (PAP) procedures. J Histochem Cytochem 29: 577-580.
Hungund BL, Basavarajappa BS (2004). Role of endocannabinoids and cannabinoid $\mathrm{CB} 1$ receptors in alcohol-related behaviors. Ann N Y Acad Sci 1025: 515-527.

Hungund BL, Vinod KY, Kassir SA, Basavarajappa BS, Yalamanchili R, Cooper TB et al (2004). Upregulation of CB1 receptors and agonist-stimulated [35S]GTPgammaS binding in the prefrontal cortex of depressed suicide victims. Mol Psychiatry 9: 184-190.

Katona I, Rancz EA, Acsady L, Ledent C, Mackie K, Hajos N et al (2001). Distribution of CB1 cannabinoid receptors in the amygdala and their role in the control of GABAergic transmission. J Neurosci 21: 9506-9518.

Katona I, Sperlagh B, Magloczky Z, Santha E, Kofalvi A, Czirjak S et al (2000). GABAergic interneurons are the targets of cannabinoid actions in the human hippocampus. Neuroscience 100: 797-804.

Katona I, Sperlagh B, Sik A, Kafalvi A, Vizi ES, Mackie K et al (1999). Presynaptically located CB1 cannabinoid receptors regulate GABA release from axon terminals of specific hippocampal interneurons. J Neurosci 19: 4544-4558.

Katona I, Urban GM, Wallace M, Ledent C, Jung KM, Piomelli D et al (2006). Molecular composition of the endocannabinoid system at glutamatergic synapses. J Neurosci 26: 5628-5637.

Kawamura Y, Fukaya M, Maejima T, Yoshida T, Miura E, Watanabe $\mathrm{M}$ et al (2006). The CB1 cannabinoid receptor is the major cannabinoid receptor at excitatory presynaptic sites in the hippocampus and cerebellum. J Neurosci 26: 2991-3001.

Kim J, Alger BE (2004). Inhibition of cyclooxygenase-2 potentiates retrograde endocannabinoid effects in hippocampus. Nat Neurosci 7: 697-698.

Koethe D, Llenos IC, Dulay JR, Hoyer C, Torrey EF, Leweke FM et al (2007). Expression of CB1 cannabinoid receptor in the anterior cingulate cortex in schizophrenia, bipolar disorder, and major depression. J Neural Transm 114: 1055-1063.

Leweke FM, Giuffrida A, Wurster U, Emrich HM, Piomelli D (1999). Elevated endogenous cannabinoids in schizophrenia. Neuroreport 10: 1665-1669.

Lewis DA, Hashimoto T, Volk DW (2005). Cortical inhibitory neurons and schizophrenia. Nat Rev Neurosci 6: 312-324.

Lewis DA, Sweet RA (2009). Schizophrenia from a neural circuitry perspective: advancing toward rational pharmacological therapies. J Clin Invest 119: 706-716.

Linszen DH, Dingemans PM, Lenior ME (1994). Cannabis abuse and the course of recent-onset schizophrenic disorders. Arch Gen Psychiatry 51: 273-279.

Makara JK, Mor M, Fegley D, Szabo SI, Kathuria S, Astarita G et al (2005). Selective inhibition of 2-AG hydrolysis enhances endocannabinoid signaling in hippocampus. Nat Neurosci 8: 1139-1141.

Mangieri RA, Piomelli D (2007). Enhancement of endocannabinoid signaling and the pharmacotherapy of depression. Pharmacol Res 56: 360-366.

Marco EM, Granstrem O, Moreno E, Llorente R, Adriani W, Laviola G et al (2007). Subchronic nicotine exposure in adolescence induces long-term effects on hippocampal and striatal cannabinoid-CB1 and mu-opioid receptors in rats. Eur J Pharmacol 557: 37-43.

Marsicano G, Lutz B (1999). Expression of the cannabinoid receptor $\mathrm{CB} 1$ in distinct neuronal subpopulations in the adult mouse forebrain. Eur J Neurosci 11: 4213-4225.

Morozov YM, Freund TF (2003). Post-natal development of type 1 cannabinoid receptor immunoreactivity in the rat hippocampus. Eur J Neurosci 18: 1213-1222.

Morris HM, Lewis DA (2009). GABA-related mRNA expression in the dorsolateral prefrontal cortex (DLPFC) of subjects with bipolar or major depressive disorder. Schizophr Bull 35: 231-232.

Murray RM, Morrison PD, Henquet C, Di Forti M (2007). Cannabis, the mind and society: the hash realities. Nat Rev Neurosci 8: 885-895. 
Newell KA, Deng C, Huang XF (2006). Increased cannabinoid receptor density in the posterior cingulate cortex in schizophrenia. Exp Brain Res 172: 556-560.

Oeth KM, Lewis DA (1993). Postnatal development of the cholecystokinin innervation of monkey prefrontal cortex. J Comp Neurol 336: 400-418.

Ohno-Shosaku T, Tsubokawa H, Mizushima I, Yoneda N, Zimmer A, Kano M (2002). Presynaptic cannabinoid sensitivity is a major determinant of depolarization-induced retrograde suppression at hippocampal synapses. J Neurosci 22: 3864-3872.

Pierri JN, Chaudry AS, Woo T-U, Lewis DA (1999). Alterations in chandelier neuron axon terminals in the prefrontal cortex of schizophrenic subjects. Am J Psychiatry 156: 1709-1719.

Pistis M, Ferraro L, Pira L, Flore G, Tanganelli S, Gessa GL et al (2002). Delta(9)-tetrahydrocannabinol decreases extracellular GABA and increases extracellular glutamate and dopamine levels in the rat prefrontal cortex: an in vivo microdialysis study. Brain Res 948: 155-158.

Price MR, Baillie GL, Thomas A, Stevenson LA, Easson M, Goodwin $\mathrm{R}$ et al (2005). Allosteric modulation of the cannabinoid CB1 receptor. Mol Pharmacol 68: 1484-1495.

Rajkowska G, Goldman-Rakic PS (1995). Cytoarchitectonic definition of prefrontal areas in the normal human cortex: I. Remapping of areas 9 and 46 using quantitative criteria. Cereb Cortex 5: 307-322.

Rao SG, Williams GV, Goldman-Rakic PS (2000). Destruction and creation of spatial tuning by disinhibition: $\mathrm{GABA}_{\mathrm{A}}$ blockade of prefrontal cortical neurons engaged by working memory. J Neurosci 20: 485-494.

Reichenberg A, Caspi A, Harrington H, Houts R, Keefe RS, Murray $\mathrm{RM}$ et al (2010). Static and dynamic cognitive deficits in childhood preceding adult schizophrenia: a 30-year study. Am J Psychiatry 167: 160-169.

Rimondini R, Arlinde C, Sommer W, Heilig M (2002). Long-lasting increase in voluntary ethanol consumption and transcriptional regulation in the rat brain after intermittent exposure to alcohol. FASEB J 16: 27-35.

Romero J, Berrendero F, Manzanares J, Perez A, Corchero J, Fuentes JA et al (1998). Time-course of the cannabinoid receptor down-regulation in the adult rat brain caused by repeated exposure to delta9-tetrahydrocannabinol. Synapse 30: 298-308.

Romero J, Garcia L, Fernandez-Ruiz JJ, Cebeira M, Ramos JA (1995). Changes in rat brain cannabinoid binding sites after acute or chronic exposure to their endogenous agonist, anandamide, or to delta 9-tetrahydrocannabinol. Pharmacol Biochem Behav 51: 731-737.

Romero J, Garcia-Palomero E, Castro JG, Garcia-Gil L, Ramos JA, Fernandez-Ruiz JJ (1997). Effects of chronic exposure to delta9tetrahydrocannabinol on cannabinoid receptor binding and mRNA levels in several rat brain regions. Brain Res Mol Brain Res 46: 100-108.

Rubino T, Massi P, Patrini G, Venier I, Giagnoni G, Parolaro D (1994). Chronic CP-55,940 alters cannabinoid receptor mRNA in the rat brain: an in situ hybridization study. Neuroreport 5: 2493-2496.

Sawaguchi T, Matsumura M, Kubota K (1988). Delayed response deficit in monkeys by locally disturbed prefrontal neuronal activity by bicuculline. Behav Brain Res 31: 193-198.
Sim-Selley LJ (2003). Regulation of cannabinoid CB1 receptors in the central nervous system by chronic cannabinoids. Crit Rev Neurobiol 15: 91-119.

Sundram S, Copolov D, Dean B (2005). Clozapine decreases [3H] CP 55940 binding to the cannabinoid 1 receptor in the rat nucleus accumbens. Naunyn Schmiedebergs Arch Pharmacol 371: 428-433.

Sweet RA, Henteleff RA, Zhang W, Sampson AR, Lewis DA (2009). Reduced dendritic spine density in auditory cortex of subjects with schizophrenia. Neuropsychopharmacology 34: 374-389.

Terry GE, Hirvonen J, Liow JS, Zoghbi SS, Gladding R, Tauscher JT et al (2010). Imaging and quantitation of cannabinoid CB1 receptors in human and monkey brains using (18)F-labeled inverse agonist radioligands. J Nucl Med 51: 112-120.

Trettel J, Fortin DA, Levine ES (2004). Endocannabinoid signalling selectively targets perisomatic inhibitory inputs to pyramidal neurones in juvenile mouse neocortex. J Physiol 556: 95-107.

Uriguen L, Garcia-Fuster MJ, Callado LF, Morentin B, La Harpe R, Casado $\mathrm{V}$ et al (2009). Immunodensity and mRNA expression of A2A adenosine, D2 dopamine, and CB1 cannabinoid receptors in postmortem frontal cortex of subjects with schizophrenia: effect of antipsychotic treatment. Psychopharmacology (Berl) 206: 313-324.

Vinod KY, Arango V, Xie S, Kassir SA, Mann JJ, Cooper TB et al (2005). Elevated levels of endocannabinoids and CB1 receptormediated G-protein signaling in the prefrontal cortex of alcoholic suicide victims. Biol Psychiatry 57: 480-486.

Volavka J, Cooper T, Czobor P, Bitter I, Meisner M, Laska E et al (1992). Haloperidol blood levels and clinical effects. Arch Gen Psychiatry 49: 354-361.

Volk DW, Pierri JN, Fritschy J-M, Auh S, Sampson AR, Lewis DA (2002). Reciprocal alterations in pre- and postsynaptic inhibitory markers at chandelier cell inputs to pyramidal neurons in schizophrenia. Cereb Cortex 12: 1063-1070.

Weinberger DR, Egan MF, Bertolino A, Callicott JH, Mattay VS, Lipska BK et al (2001). Prefrontal neurons and the genetics of schizophrenia. Biol Psychiatry 50: 825-844.

Westlake TM, Howlett AC, Ali SF, Paule MG, Scallet AC, Slikker Jr W (1991). Chronic exposure to delta 9-tetrahydrocannabinol fails to irreversibly alter brain cannabinoid receptors. Brain Res 544: 145-149.

Wiley JL, Kendler SH, Burston JJ, Howard DR, Selley DE, SimSelley LJ (2008). Antipsychotic-induced alterations in CB1 receptor-mediated G-protein signaling and in vivo pharmacology in rats. Neuropharmacology 55: 1183-1190.

Yasuno F, Brown AK, Zoghbi SS, Krushinski JH, Chernet E, Tauscher $\mathrm{J}$ et al (2008). The PET radioligand [11C]MePPEP binds reversibly and with high specific signal to cannabinoid CB1 receptors in nonhuman primate brain. Neuropsychopharmacology 33: 259-269.

Zavitsanou K, Garrick T, Huang XF (2004). Selective antagonist [3H]SR141716A binding to cannabinoid CB1 receptors is increased in the anterior cingulate cortex in schizophrenia. Prog Neuropsychopharmacol Biol Psychiatry 28: 355-360.

Zhuang S, Kittler J, Grigorenko EV, Kirby MT, Sim LJ, Hampson $\mathrm{RE}$ et al (1998). Effects of long-term exposure to delta9-THC on expression of cannabinoid receptor (CB1) mRNA in different rat brain regions. Brain Res Mol Brain Res 62: 141-149.

Supplementary Information accompanies the paper on the Neuropsychopharmacology website (http://www.nature.com/npp) 